\title{
JACOB'S LADDERS, THE STRUCTURE OF THE HARDY-LITTLEWOOD INTEGRAL AND SOME NEW CLASS OF NONLINEAR INTEGRAL EQUATIONS
}

\author{
JAN MOSER
}

\begin{abstract}
In this paper we obtain new formulae for short and microscopic parts of the Hardy-Littlewood integral, and the first asymptotic formula for the sixth order expression $\left|\zeta\left(\frac{1}{2}+i \varphi_{1}(t)\right)\right|^{4}\left|\zeta\left(\frac{1}{2}+i t\right)\right|^{2}$. These formulae cannot be obtained in the theories of Balasubramanian, Heath-Brown and Ivic.

Dedicated to the 75th aniversary of Anatolii Alekseevich Karatsuba.
\end{abstract}

\section{INTRODUCTION}

Let us remind that Hardy and Littlewood started to study the following integral in 1918

$$
\int_{0}^{T}\left|\zeta\left(\frac{1}{2}+i t\right)\right|^{2} \mathrm{~d} t=\int_{0}^{T} Z^{2}(t) \mathrm{d} t
$$

where

$$
Z(t)=e^{i \vartheta(t)} \zeta\left(\frac{1}{2}+i t\right), \vartheta(t)=-\frac{t}{2} \ln \pi+\operatorname{Im} \ln \Gamma\left(\frac{1}{4}+i \frac{t}{2}\right),
$$

and they have derived the following formula ([2], pp. 122, 151-156)

$$
\int_{0}^{T} Z^{2}(t) \mathrm{d} t \sim T \ln T, T \rightarrow \infty .
$$

We have shown in the paper 8 , that except the asymptotic formula (1.3) possessing an unbounded error there is an infinite family of other asymptotic representations of the Hardy-Littlewood integral (1.1). Each member of this family is an almost exact representation of the integral (1.1). Namely, the following formula

$$
\int_{0}^{T} Z^{2}(t) \mathrm{d} t=\frac{\varphi(T)}{2} \ln \frac{\varphi(T)}{2}+(c-\ln 2 \pi) \frac{\varphi(T)}{2}+c_{0}+\mathcal{O}\left(\frac{\ln T}{T}\right)
$$

takes place where $\varphi(T)$ is the Jacob's ladder, i.e. an arbitrary solution to the nonlinear integral equation

$$
\int_{0}^{\mu[x(T)]} Z^{2}(t) e^{-\frac{2}{x(T)} t} \mathrm{~d} t=\int_{0}^{T} Z^{2}(t) \mathrm{d} t
$$

Key words and phrases. Riemann zeta-function. 
and $\mu(y) \geq 7 y \ln y ; \mu(y) \rightarrow y=\varphi_{\mu}(T)=\varphi(T)$.

We obtain new properties of the signal (1.2) generated by the Riemann zetafunction. Namely:

(A) We obtain the multiplicative asymptotic formula

$$
\int_{T}^{T+U} Z^{2}(t) \mathrm{d} t \sim U \ln T \tan [\alpha(T, U)], U \in\left(0, \frac{T}{\ln T}\right], T \rightarrow \infty,
$$

in the parts 2-7 of this paper. The application on microscopic $(0<U<$ 1) and short $\left(1 \leq U<T^{1 / 3+2 \epsilon}\right)$ parts of the Hardy-Littlewood integral (1.1) is the main aim of this formula.

(B) We also obtain, in the parts 8 - 10 of this work, the formula

$$
\int_{T}^{T+U_{1}}\left|\zeta\left(\frac{1}{2}+i \varphi_{1}(t)\right)\right|^{4}\left|\zeta\left(\frac{1}{2}+i t\right)\right|^{2} \mathrm{~d} t \sim \frac{1}{2 \pi} U_{1} \ln ^{5} T, T \rightarrow \infty
$$

where $U_{1}=T^{7 / 8+2 \epsilon}, \varphi_{1}(t)=\frac{1}{2} \varphi(t)$. This formula is the first integral asymptotic formula in the theory of the Riemann zeta-function for the sixth-order expression $\left|\zeta\left(\frac{1}{2}+i \varphi_{1}(t)\right)\right|^{4}\left|\zeta\left(\frac{1}{2}+i t\right)\right|^{2}$.

(C) In the part 11 of this work the following property, for example, is noticed: the Jacob's ladder $\varphi_{1}$ is the asymptotic solution of the nonlinear integral equation

$$
\int_{x^{-1}(T)}^{x^{-1}(T+2)} \frac{\left[\mathcal{T}_{n}(x(t)-T-1)\right]^{2}}{\sqrt{1-(x(t)-T-1)^{2}}}\left|\zeta\left(\frac{1}{2}+i t\right)\right|^{2} \mathrm{~d} t=\frac{\pi}{2} \ln T,
$$

$n=1,2, \ldots$, where $\mathcal{T}_{n}(t), t \in[-1,1]$ is the Chebyshev polynomial of the first kind, i.e. the following asymptotic formula

$\int_{\varphi_{1}^{-1}(T)}^{\varphi_{1}^{-1}(T+2)} \frac{\left[\mathcal{T}_{n}\left(\varphi_{1}(t)-T-1\right)\right]^{2}}{\sqrt{1-\left(\varphi_{1}(t)-T-1\right)^{2}}}\left|\zeta\left(\frac{1}{2}+i t\right)\right|^{2} \mathrm{~d} t \sim \frac{\pi}{2} \ln T, T \rightarrow \infty$

holds true.

2. NeCESSity OF A NEW EXPRESSION FOR THE SHORT AND THE MICROSCOPIC PARTS OF THE HARDY-LITTLEWOOD INTEGRAL

The Balasubramanian's formula

$$
\int_{0}^{T} Z^{2}(t) \mathrm{d} t=T \ln T+(2 c-1-\ln 2 \pi) T+\mathcal{O}\left(T^{1 / 3+\epsilon}\right)
$$

implies (comp. [8], (2.5), (8.3))

$$
\int_{T}^{T+U_{0}} Z^{2}(t) \mathrm{d} t=U_{0} \ln T+(2 c-\ln 2 \pi) U_{0}+\mathcal{O}\left(T^{1 / 3+\epsilon}\right), U_{0}=T^{1 / 3+2 \epsilon}
$$

where $c$ is the Euler's constant. Furthermore, let us remind the Heath-Brown's estimate (see [5], (7.20), p. 178)

Page 2 of 16 


$$
\begin{aligned}
& \int_{T-G}^{T+G} Z^{2}(t) \mathrm{d} t= \\
& \mathcal{O}\left\{G \ln T+G \sum_{K}(T K)^{-1 / 2}\left(S(K)+K^{-1} \int_{0}^{K}|S(x)| \mathrm{d} x\right) e^{-G^{2} K / T}\right\}
\end{aligned}
$$

(for the definition of used symbols see [5], (7.21)-(7.23)), uniformly for $T^{\epsilon} \leq G \leq$ $T^{1 / 2+\epsilon}$. And, finally, we add the Ivic' estimate $([5],(7.62))$

$$
\int_{T-G}^{T+G} Z^{2}(t) \mathrm{d} t=\mathcal{O}\left(G \ln ^{2} T\right), G \geq T^{1 / 3-\epsilon_{0}}, \epsilon_{0}=\frac{1}{108} \approx 0.009 .
$$

Remark 1. It is obvious that the short intervals $[T-G, T+G]$ with $G=1000$, for example, are not included in the methods od Balasubramanian, Heath-Brown and Ivic, as well, leading to (2.1)-(2.3).

In this paper we present a new method how to deal with short and microscopic parts

$$
\int_{T}^{T+U} Z^{2}(t) \mathrm{d} t
$$

of the Hardy-Littlewood integral (1.1). In order to attain this goal we will use only elementary geometric properties of the Jacob's ladders. The basic idea is expressed in the following theorem.

Theorem 1. For $\mu[\varphi]=a \varphi \ln \varphi, a \in[7,8]$ the following is true

$$
\begin{aligned}
& \int_{T}^{T+U} Z^{2}(t) \mathrm{d} t=\left\{1+\mathcal{O}\left(\frac{\ln \ln T}{\ln T}\right)\right\} U \ln T \tan [\alpha(T, U)], \\
& U \in\left(0, \frac{T}{\ln T}\right], T \geq T_{0}[\varphi]
\end{aligned}
$$

where $\alpha=\alpha(T, U)$ is the angle of the chord of the curve $y=\frac{1}{2} \varphi(T)$ that binds the points $\left[T, \frac{1}{2} \varphi(T)\right]$ and $\left[T+U, \frac{1}{2} \varphi(T+U)\right]$.

\section{A GEOMETRIC CRITERION FOR VALIDITy of the USUAL MEAN-VALUe} THEOREM

3.1. First of all we will show the canonical equivalence that follows from (2.5). Let us remind (see 8 , (8.3)) that we call the chord binding the points

$$
\left[T, \frac{1}{2} \varphi(T)\right] ;\left[T+U_{0}, \frac{1}{2} \varphi\left(T+U_{0}\right)\right]
$$

of the Jacob's ladder $y=\frac{1}{2} \varphi(T)$ the fundamental chord. By comparison of the formulae (2.1) and (2.5), $U=U_{0}$, we obtain the following asymptotic formula

$$
\tan \left[\alpha\left(T, U_{0}\right)\right]=\frac{\varphi\left(T+U_{0}\right)-\varphi(T)}{2 U_{0}}=1+\mathcal{O}\left(\frac{\ln \ln T}{\ln T}\right) .
$$


Definition. The chord binding the points

$$
\left[N, \frac{1}{2} \varphi(N)\right] ;\left[M, \frac{1}{2} \varphi(M)\right],[N, M] \subset\left[T, T+U_{0}\right]
$$

which fulfills the property

$$
\tan [\alpha(N, M-N)]=1+o(1), T \rightarrow \infty
$$

(compare (3.2)) is called the almost parallel chord to the fundamental chord. This property will be denoted by the symbol $/ 7$.

Corollary 1. Let $[N, M] \subset\left[T, T+U_{0}\right]$. Then

$$
\frac{1}{M-N} \int_{N}^{M} Z^{2}(t) \mathrm{d} t \sim \ln T \quad \Leftrightarrow \quad \not \text {. }
$$

Remark 2. Wee see that the analytic property

$$
\frac{1}{M-N} \int_{N}^{M} Z^{2}(t) \mathrm{d} t \sim \ln T
$$

(the usual mean-value theorem) is equivalent to the geometric property $\square$ of the Jacob's ladder $y=\frac{1}{2} \varphi(T)$.

3.2. Let us consider the set of all chords of the curve $y=\frac{1}{2} \varphi(T)$ which are almost parallel to the fundamental chord. Let the generic chord of this set bind the points (3.3). Then, from Corollary 1, we obtain

Corollary 2. There is an continuum of intervals $[N, M] \subset\left[T, T+U_{0}\right]$ such that the following asymptotic formula

$$
\int_{N}^{M} Z^{2}(t) \mathrm{d} t \sim(M-N) \ln T
$$

holds true.

Remark 3. There is, for example, continuum of intervals $[N, M]: 0<M-N<1$ such that the asymptotic formula (3.5) holds true (chords approaching to zero).

\section{ON THE MicRoscopic PARTS OF THE HARDY-LitTlEWOOd INTEGRAL IN THE NEIGHBOURHOODS OF ZEROES OF THE FUNCTION $\zeta\left(\frac{1}{2}+i t\right)$}

Let $\gamma, \gamma^{\prime}$ be a pair of neighbouring zeroes of the function $\zeta\left(\frac{1}{2}+i t\right)$. The function $\frac{1}{2} \varphi(T)$ is necessarily convex on some right neighbourhood of the point $T=\gamma$, and this function is necessarily concave on some left neighbourhood of the point $T=\gamma^{\prime}$. Therefore, there exists the minimal value $\rho \in\left(\gamma, \gamma^{\prime}\right)$ such that $\left[\rho, \frac{1}{2} \varphi(\rho)\right]$ is the inflexion point of the curve $y=\frac{1}{2} \varphi(t)$. At this point, by properties of the Jacob's ladders, we have $\varphi^{\prime}(\rho)>0$. Let furthermore $\beta=\beta(\gamma, \rho)$ be the angle of the chord binding the points

$$
\left[\gamma, \frac{1}{2} \varphi(\gamma)\right] ;\left[\rho, \frac{1}{2} \varphi(\rho)\right]
$$

Then we obtain from Theorem 1

Corollary 3. For every sufficiently big zero $T=\gamma$ of the function $\zeta\left(1+\frac{1}{2} i T\right)$ the following formulae describing the microscopic parts of the Hardy-Littlewood integral hold true

Page 4 of 16 
(A) continuum of formulae

$$
\int_{\gamma}^{\gamma+U} Z^{2}(t) \mathrm{d} t \sim U \ln \gamma \tan \alpha, \alpha \in(0, \beta(\gamma, \rho)), U=U(\gamma, \alpha) \in(0, \rho-\gamma)
$$

where $\alpha$ is the angle of the rotating chord binding the points $\left[\gamma, \frac{1}{2} \varphi(\gamma)\right]$, $\left[\gamma+U, \frac{1}{2} \varphi(\gamma+U)\right]$

(B) continuum of the formulae for the chords parallel to the chord given by the points (4.1)

$$
\int_{N}^{M} Z^{2}(t) \mathrm{d} t \sim(M-N) \ln N \tan [\beta(\gamma, \rho)], \quad \gamma \leq N<M<\gamma^{\prime} .
$$

Remark 4. The notion microscopic parts of the Hardy-Littlewood integral has its natural origin in the following: by Karatsuba's selbergian estimate (see [6], p. 265) for almost all intervals $\left[\gamma, \gamma^{\prime}\right] \subset\left[T, T+T^{1 / 3+2 \epsilon}\right]$ we have

$$
\gamma^{\prime}-\gamma<A \frac{\ln \ln T}{\ln T} \rightarrow 0, T \rightarrow \infty .
$$

Remark 5. In connection with (4.4) we can remind that if the Riemann hypothesis is true then the Littlewood's estimate takes place (see [7])

$$
\gamma^{\prime}-\gamma<\frac{A}{\ln \ln \gamma}, \gamma \rightarrow \infty
$$

\section{SECOND Class of Formulae for PARTS of the HARdy-LitTlewood INTEGRAL BEGINNING IN ZEROES OF THE FUNCTION $\zeta\left(\frac{1}{2}+i t\right)$}

Let $\gamma, \bar{\gamma}$ be a pair of zeroes of the function $\zeta\left(\frac{1}{2}+i t\right)$ such that $\bar{\gamma}$ obeys the following conditions

$$
\bar{\gamma}=\gamma+\gamma^{1 / 3+2 \epsilon}+\Delta(\gamma), 0 \leq \Delta(\gamma)=\mathcal{O}\left(\gamma^{1 / 4+\epsilon}\right)
$$

(it is sufficient to use the classical Hardy-Littlewood's estimate for the distance between the neighbouring zeroes, [2], pp. 125, 177-184). Consequently

$$
U(\gamma)=\gamma^{1 / 3+2 \epsilon}+\Delta(\gamma) \sim \gamma^{1 / 3+2 \epsilon}, \gamma \rightarrow \infty
$$

For the chord binding the points

$$
\left[\gamma, \frac{1}{2} \varphi(\gamma)\right],\left[\bar{\gamma}, \frac{1}{2} \varphi(\bar{\gamma})\right]
$$

we have by (3.1), (5.1)

$$
\tan [\alpha(\gamma, U(\gamma))]=1+\mathcal{O}\left(\frac{1}{\ln \gamma}\right)
$$

The continuous curve $y=\frac{1}{2} \varphi(T)$ lies bellow the chord given by the points (5.2) on some right neighbourhood of the point $T=\gamma$, and this curve lies above that chord on some left neighbourhood of the point $T=\bar{\gamma}$. Therefore, there exists a common point of the curve and of the chord. Let $\left[\bar{\rho}, \frac{1}{2} \varphi(\bar{\rho})\right], \bar{\rho} \in(\gamma, \bar{\gamma})$ be such a common point that is the closest one to the point $\left[\gamma, \frac{1}{2} \varphi(\gamma)\right]$. Then we obtain from Theorem 1 the following

Page 5 of 16 
Corollary 4. For every sufficient big zero $T=\gamma$ of the function $\zeta\left(\frac{1}{2}+i T\right)$ we have the following formulae for the parts (2.4) of the Hardy-Littlewood integral (1.1)

(A) continuum of formulae for the rotating chord

$$
\int_{\gamma}^{\gamma+U} Z^{2}(t) \mathrm{d} t \sim U \ln \gamma \tan \alpha, \alpha \in[\eta, 1-\eta], U=U(\gamma, \alpha) \in(0, \bar{\rho}-\gamma)
$$

where $\alpha=\alpha(\gamma, U)$ is the angle of the rotating chord binding the points $\left[\gamma, \frac{1}{2} \varphi(\gamma)\right]$ and $\left[\gamma+U, \frac{1}{2} \varphi(\gamma+U)\right]$, and $0<\eta$ is an arbitrarily small number,

(B) continuum of formulae for the chords parallel (and almost parallel) to the chord binding the points (5.2)

$$
\int_{N}^{M} Z^{2}(t) \mathrm{d} t \sim(M-N) \ln N, \gamma \leq N<M \leq \bar{\gamma}
$$

Remark 6. For example, in the case $\alpha=\pi / 6$ we have from (5.4)

$$
\int_{\gamma}^{\gamma+U(\gamma, \pi / 6)} Z^{2}(t) \mathrm{d} t \sim \frac{1}{\sqrt{3}} U \ln \gamma
$$

for every sufficiently big zero $T=\gamma$ of the function $\zeta\left(\frac{1}{2}+i T\right)$.

Remark 7. It is clear that the asymptotic formulae (2.5), (3.4), (4.2), (4.3), (5.4) and (5.5) cannot be derived within complicated methods of Balasubramanian, HeathBrown and Ivic.

\section{An estimate FOR $\Phi_{\varphi \varphi}^{\prime \prime}[\varphi(T)]$}

Let us remind (see $[8],(3.5),(3.9))$ that

$$
Z^{2}(t)=\Phi_{\varphi}^{\prime}[\varphi(t)] \frac{\mathrm{d} \varphi(t)}{\mathrm{d} t},
$$

where

$$
\Phi_{\varphi}^{\prime}[\varphi]=\frac{2}{\varphi^{2}} \int_{0}^{\mu[\varphi]} t e^{-\frac{2}{\varphi} t} Z^{2}(t) \mathrm{d} t+Z^{2}\{\mu[\varphi]\} e^{-\frac{2}{\varphi} \mu[\varphi]} \frac{\mathrm{d} \mu(\varphi)}{\mathrm{d} \varphi} .
$$

The following lemma is true.

Lemma 1. If $\mu[\varphi]=a \varphi \ln \varphi, a \in[7,8]$ then

$$
\Phi_{\varphi \varphi}^{\prime \prime}[\varphi(T)]=\mathcal{O}\left(\frac{1}{\varphi} \ln \varphi \ln \ln \varphi\right), T \geq T_{0}[\varphi]
$$

uniformly with respect to a.

Remark 8 . The segment $[7,8]$ is sufficient to our purpose since the continuum of Jacob's ladders corresponds to this segment.

Proof. First of all we have (see (6.2) $)$

$$
\Phi_{\varphi \varphi}^{\prime \prime}[\varphi]=\frac{4}{\varphi^{3}} \int_{0}^{\mu[\varphi]} t\left(\frac{t}{\varphi}-1\right) e^{-\frac{2}{\varphi} t} Z^{2}(t) \mathrm{d} t+Q[\varphi],
$$




$$
\begin{aligned}
& Q[\varphi]=e^{-\frac{2}{\varphi} \mu[\varphi]}\left\{\frac{4}{\varphi^{2}} Z^{2}\{\mu[\varphi]\} \mu[\varphi] \frac{\mathrm{d} \mu[\varphi]}{\mathrm{d} \varphi}-\frac{2}{\varphi} Z^{2}\{\mu[\varphi]\}\left(\frac{\mathrm{d} \mu[\varphi]}{\mathrm{d} \varphi}\right)^{2}+\right. \\
& \left.2 Z\{\mu[\varphi]\} Z_{\mu}^{\prime}\{\mu[\varphi]\}\left(\frac{\mathrm{d} \mu[\varphi]}{\mathrm{d} \varphi}\right)^{2}+Z^{2}\{\mu[\varphi]\} \frac{\mathrm{d}^{2} \mu[\varphi]}{\mathrm{d} \varphi^{2}}\right\}
\end{aligned}
$$

Let

$$
g(t)=t\left(\frac{t}{\varphi}-1\right) e^{-\frac{2}{\varphi} t}, t \in[0, \mu[\varphi]]
$$

We apply the following elementary facts

$$
\begin{aligned}
& g(0)=g(\varphi)=0, g^{\prime}\left[\left(1-\frac{1}{\sqrt{2}}\right) \varphi\right]=g^{\prime}\left[\left(1+\frac{1}{\sqrt{2}}\right) \varphi\right]=0, \\
& \min \{g(t)\}=-\frac{1}{\sqrt{2}}\left(1-\frac{1}{\sqrt{2}}\right) e^{-2+\sqrt{2}} \varphi \\
& \max \{g(t)\}=\frac{1}{\sqrt{2}}\left(1+\frac{1}{\sqrt{2}}\right) e^{-2-\sqrt{2}} \varphi, \\
& g(t) \leq g(\varphi \ln \ln \varphi)<\varphi\left(\frac{\ln \ln \varphi}{\ln \varphi}\right)^{2}, t \in[\varphi \ln \ln \varphi, 8 \varphi \ln \varphi], \\
& Z(t), Z^{\prime}(t)=\mathcal{O}\left(t^{1 / 4}\right),
\end{aligned}
$$

and the Hardy-Littlewood formula (1.3). We have

$$
\begin{aligned}
& \frac{4}{\varphi^{3}} \int_{0}^{\varphi \ln \ln \varphi}=\mathcal{O}\left(\frac{1}{\varphi^{2}} \int_{0}^{\varphi \ln \ln \varphi} Z^{2}(t) \mathrm{d} t\right)=\mathcal{O}\left(\frac{1}{\varphi} \ln \varphi \ln \ln \varphi\right), \\
& \frac{4}{\varphi^{3}} \int_{\varphi \ln \ln \varphi}^{8 \varphi \ln \varphi}=\mathcal{O}\left\{\frac{1}{\varphi^{3}} \varphi\left(\frac{\ln \ln \varphi}{\ln \varphi}\right)^{2} \varphi \ln ^{2} \varphi\right\}=\mathcal{O}\left\{\frac{1}{\varphi}(\ln \ln \varphi)^{2}\right\}
\end{aligned}
$$

by (1.3), (6.6) and (see (6.5))

$$
Q[\varphi]=\mathcal{O}\left(\varphi^{-13}\right) \rightarrow 0, T \rightarrow \infty .
$$

Finally, we obtain (6.3) from (6.4) by (6.7), (6.8).

\section{Proof of Theorem 1}

By (6.1) we have

$$
\int_{T}^{T+U} Z^{2}(t) \mathrm{d} t=\Phi_{\varphi}^{\prime}\left[\varphi\left(t_{1}\right)\right] \int_{T}^{T+U} \mathrm{~d} \varphi=\Phi_{\varphi}^{\prime}\left[\varphi\left(t_{1}\right)\right]\{\varphi(T+U)-\varphi(T)\},
$$

i.e.

$$
\begin{aligned}
& \int_{T}^{T+U} Z^{2}(t) \mathrm{d} t=2 U \Phi_{\varphi}^{\prime}\left[\varphi\left(t_{1}\right)\right] \tan [\alpha(T, U)], t_{1}=t_{1}(U) \in(T, T+U), \\
& \tan [\alpha(T, U)]=\frac{\varphi(T+U)-\varphi(T)}{2 U} .
\end{aligned}
$$

Next, we have 


$$
\int_{T}^{T+U_{0}} Z^{2}(t) \mathrm{d} t=2 U_{0} \Phi_{\varphi}\left[\varphi\left(t_{2}\right)\right]\left\{1+\mathcal{O}\left(\frac{1}{\ln T}\right)\right\}, t_{2}=t_{2}\left(U_{0}\right) \in\left(T, T+U_{0}\right),
$$

by (3.2), (7.1). Hence, by the comparison of the formulae (2.1) and (7.2) we obtain

$$
\Phi_{\varphi}^{\prime}\left[\varphi\left(t_{2}\right)\right]=\frac{1}{2} \ln T+\mathcal{O}(1) .
$$

Next, from the formula (see $[8],(6.2)$ )

$$
T-\frac{\varphi(T)}{2} \sim(1-c) \pi(T) ; T \sim \frac{\varphi(T)}{2},
$$

we obtain

$$
\varphi\left(t_{1}\right)-\varphi\left(t_{2}\right)=2\left(t_{1}-t_{2}\right)+\mathcal{O}\left(\frac{T}{\ln T}\right)=\mathcal{O}\left(\frac{T}{\ln T}\right), U \in\left(0, \frac{T}{\ln T}\right],
$$

and subsequently (see (6.3)

$$
\Phi_{\varphi}^{\prime}\left[\varphi\left(t_{1}\right)\right]-\Phi_{\varphi}^{\prime}\left[\varphi\left(t_{2}\right)\right]=\mathcal{O}\left\{\left|\Phi_{\varphi \varphi}^{\prime \prime}(T)\right| \cdot\left|\varphi\left(t_{1}\right)-\varphi\left(t_{2}\right)\right|\right\}=\mathcal{O}(\ln \ln T) .
$$

Therefore we obtain

$$
\Phi_{\varphi}^{\prime}\left[\varphi\left(t_{1}\right)\right]=\frac{1}{2} \ln T+\mathcal{O}(\ln \ln T),
$$

by (7.3), (7.6). Finally, (2.5) follows from (7.1), (7.7).

Remark 9. Similarly to (7.6) we have

$$
\Phi_{\varphi}^{\prime}\left[\varphi\left(t_{1}\right)\right]-\Phi_{\varphi}^{\prime}[\varphi(t)]=\mathcal{O}(\ln \ln T), t \in[T, T+U],
$$

and obtain (see (6.1), (7.7), (7.8) )

$$
Z^{2}(t)=\frac{1}{2}\left\{1+\mathcal{O}\left(\frac{\ln \ln t}{\ln t}\right)\right\} \ln t \frac{\mathrm{d} \varphi(t)}{\mathrm{d} t}, t \in[T, T+U], U \in\left(0, \frac{T}{\ln T}\right] .
$$

8. The integral asymptotic Formula that CONTAins the EXPRESSiOn of THE SIXTH ORDER $\left|\zeta\left(\frac{1}{2}+i \varphi_{1}(t)\right)\right|^{4}\left|\zeta\left(\frac{1}{2}+i t\right)\right|^{2}$

8.1. Let us remind that Hardy and Littlewood started to study the following integral in 1926

$$
\int_{1}^{T}\left|\zeta\left(\frac{1}{2}+i t\right)\right|^{4} \mathrm{~d} t=\int_{1}^{T} Z^{4}(t) \mathrm{d} t
$$

and they derived the following estimate (see [3], pp. 41,59; [10], p. 124)

$$
\int_{1}^{T}\left|\zeta\left(\frac{1}{2}+i t\right)\right|^{4} \mathrm{~d} t=\mathcal{O}\left(T \ln ^{4} T\right)
$$

In 1926 Ingham derived the asymptotic formula 


$$
\int_{1}^{T}\left|\zeta\left(\frac{1}{2}+i t\right)\right|^{4} \mathrm{~d} t=\frac{1}{2 \pi^{2}} T \ln ^{4} T+\mathcal{O}\left(T \ln ^{3} T\right)
$$

(see 4, p. 277, 10, p. 129). Let us remind, finally, the Ingham - Heath-Brown formula (see [5, p. 129)

$$
\int_{0}^{T} Z^{4}(t) \mathrm{d} t=T \sum_{k=0}^{4} C_{k} \ln ^{4-k} T+\mathcal{O}\left(T^{7 / 8+\epsilon}\right), C_{0}=\frac{1}{2 \pi^{2}},
$$

which improves the Ingham formula (8.1), (the small improvements of the exponents $1 / 3$ and $7 / 8$, see (2.1), (8.2) are irrelevant for our purpose).

8.2. In this direction, the following theorem holds true.

\section{Theorem 2.}

$$
\begin{aligned}
& \int_{T}^{T+U_{1}}\left|\zeta\left(\frac{1}{2}+i \varphi_{1}(t)\right)\right|^{4}\left|\zeta\left(\frac{1}{2}+i t\right)\right|^{2} \mathrm{~d} t \sim \frac{1}{2 \pi^{2}} U_{1} \ln ^{5} T, \\
& U_{1}=T^{7 / 8+2 \epsilon}, \varphi_{1}(t)=\frac{1}{2} \varphi(t), T \rightarrow \infty,
\end{aligned}
$$

and the distance of the interaction of the functions

$$
\left|\zeta\left(\frac{1}{2}+i \varphi_{1}(t)\right)\right|^{4},\left|\zeta\left(\frac{1}{2}+i t\right)\right|^{2}
$$

is

$$
t-\varphi_{1}(t) \sim(1-c) \pi(t),
$$

$c$ is the Euler's constant and $\pi(t)$ is the prime-counting function.

Remark 10. The formula (8.3) is the first integral asymptotic formula in the theory of the Riemann zeta-function for the sixth-order expression $\left|\zeta\left(\frac{1}{2}+i \varphi_{1}(t)\right)\right|^{4}\left|\zeta\left(\frac{1}{2}+i t\right)\right|^{2}$. This formula cannot be obtained by the methods of Balasubramanian, Heath-Brown and Ivic.

8.3. Since (see (8.4)

$$
T+U_{1}-\varphi_{1}\left(T+U_{1}\right) \sim(1-c) \pi\left(T+U_{1}\right), U_{1}=T^{7 / 8+2 \epsilon},
$$

we obtain

$$
T-\varphi_{1}\left(T+U_{1}\right) \sim(1-c) \pi\left(T+U_{1}\right)-U_{1} \sim(1-c) \pi(T),
$$

and consequently

$$
\rho\left\{\left[\varphi_{1}(T), \varphi_{1}(T+U)\right] ;[T, T+U]\right\} \sim(1-c) \pi(T) ; \varphi_{1}\left(T+U_{1}\right)<T
$$

where $\rho$ denotes the distance of the corresponding segments. Next, by using the mean-value theorem in (8.3), we obtain

Page 9 of 16 


\section{Corollary 5.}

$$
\left|\zeta\left(\frac{1}{2}+i \varphi_{1}(\omega)\right)\right|^{4}\left|\zeta\left(\frac{1}{2}+i \omega\right)\right|^{2} \sim \frac{1}{2 \pi^{2}} \ln ^{5} T
$$

where

$$
\omega \in\left(T, T+U_{1}\right), \varphi_{1}(\omega) \in\left(\varphi_{1}(T), \varphi_{1}\left(T+U_{1}\right)\right), \omega=\omega\left(T, U_{1}, \varphi_{1}\right) .
$$

Remark 11. Some nonlocal interaction of the functions

$$
\left|\zeta\left(\frac{1}{2}+i \varphi_{1}(t)\right)\right|^{4},\left|\zeta\left(\frac{1}{2}+i t\right)\right|^{2}
$$

is expressed by the formula (8.6). This interaction is connected with two segments unboundedly receding each from other (see (8.5); $\rho \rightarrow \infty$ as $T \rightarrow \infty$ ) - like mutually receding galaxies in the Friedman's expanding Universe.

Remark 12. Since $T \sim \omega, \omega \in\left(T, T+U_{1}\right)$ then from (8.6) we obtain

$$
\left|\zeta\left(\frac{1}{2}+i \omega\right)\right| \sim \frac{1}{\sqrt{2} \pi} \frac{\ln ^{5 / 2} \omega}{\left|\zeta\left(\frac{1}{2}+i \varphi_{1}(\omega)\right)\right|}
$$

i.e. we have the prediction of the values $|\zeta(1 / 2+i \omega)|, \omega \in(T, T+U)$ by means of the values $\left|\zeta\left(1 / 2+i \varphi_{1}(\omega)\right)\right|$ corresponding to the argument $\varphi_{1}(\omega) \in\left(\varphi_{1}(T), \varphi_{1}(T+U)\right)$ which descend from the very deep past (see (8.5), (8.7)) and - vice versa.

9. Contact point of $\left|\zeta\left(\frac{1}{2}+i t\right)\right|^{2}$ With the Class of the L-Integrable FUNCTIONS OF THE CONSTANT SIGN

Let

$$
\tilde{Z}^{2}(t)=\frac{\mathrm{d} \varphi_{1}(t)}{\mathrm{d} t}, \varphi_{1}(t)=\frac{1}{2} \varphi(t), t \geq T_{0}[\varphi]
$$

where

$$
\begin{aligned}
& \tilde{Z}^{2}(t)=\frac{Z^{2}(t)}{2 \Phi_{\varphi}^{\prime}[\varphi(t)]}=\frac{\left|\zeta\left(\frac{1}{2}+i t\right)\right|^{2}}{\left\{1+\mathcal{O}\left(\frac{\ln \ln t}{\ln t}\right)\right\} \ln t}, \\
& t \in[T, T+U], U \in\left(0, \frac{T}{\ln T}\right]
\end{aligned}
$$

(see (6.1), (7.7), (7.8)). The following lemma holds true (see (9.1))

Lemma 2. For every integrable function (in the Lebesgue sense) $f(x), x \in\left[\varphi_{1}(T), \varphi_{1}(T+\right.$ $U)$ ] the following is true

$$
\int_{T}^{T+U} f\left[\varphi_{1}(t)\right] \tilde{Z}^{2}(t) \mathrm{d} t=\int_{\varphi_{1}(T)}^{\varphi_{1}(T+U)} f(x) \mathrm{d} x, T \geq T_{0}[\varphi]
$$

where

$$
t-\varphi_{1}(t) \sim(1-c) \pi(t) .
$$

Remark 13. The formula (9.3) is true also in the case of relatively convergent improper Riemann' integral on its right-hand side.

Page 10 of 16 
If $\varphi_{1}\{[\stackrel{\circ}{T, \widehat{T+U}}]\}=[T, T+U]$ then we have the following formula (see (9.3) $)$

Lemma 3.

$$
\int_{\overparen{T}}^{\overparen{T+U}} f\left[\varphi_{1}(t)\right] \tilde{Z}^{2}(t) \mathrm{d} t=\int_{T}^{T+U} f(x) \mathrm{d} x, T \geq T_{0}[\varphi] .
$$

Next, the following lemma holds true.

Lemma 4. If $f(x) \geq 0(\leq 0), x \in\left[\varphi_{1}(T), \varphi_{1}(T+U)\right]$ then

$$
\begin{aligned}
& \int_{T}^{T+U} f\left[\varphi_{1}(t)\right]\left|\zeta\left(\frac{1}{2}+i t\right)\right|^{2} \mathrm{~d} t= \\
& =\left\{1+\mathcal{O}\left(\frac{\ln \ln T}{\ln T}\right)\right\} \ln T \int_{\varphi_{1}(T)}^{\varphi_{1}(T+U)} f(x) \mathrm{d} x, U \in\left(0, \frac{T}{\ln T}\right],
\end{aligned}
$$

and

$$
\begin{aligned}
& \int_{\stackrel{\circ}{T+U}}^{\circ} f\left[\varphi_{1}(t)\right]\left|\zeta\left(\frac{1}{2}+i t\right)\right|^{2} \mathrm{~d} t= \\
& =\left\{1+\mathcal{O}\left(\frac{\ln \ln T}{\ln T}\right)\right\} \ln T \int_{T}^{T+U} f(x) \mathrm{d} x, U \in\left(0, \frac{T}{\ln T}\right] .
\end{aligned}
$$

Proof. By using the mean-value theorem on the left-hand side of (9.3) we directly obtain (9.6), (see (9.2)). If we make use of the mean-value theorem on the left-hand side of (9.5) we obtain, by (9.2),

$$
\begin{aligned}
& \int_{\overparen{T}}^{\overparen{T+U}} f\left[\varphi_{1}(t)\right] \tilde{Z}^{2}(t) \mathrm{d} t= \\
& \frac{1}{\left\{1+\mathcal{O}\left(\frac{\ln \ln t_{1}}{\ln t_{1}}\right)\right\} \ln t_{1}} \int_{\overparen{T}}^{\overparen{T+U}} f\left[\varphi_{1}(t)\right]\left|\zeta\left(\frac{1}{2}+i t\right)\right|^{2} \mathrm{~d} t,
\end{aligned}
$$

where $t_{1} \in(\stackrel{\circ}{T, \widehat{T+U}})=\left(\varphi_{1}^{-1}(T), \varphi_{1}^{-1}(T+U)\right)$ and

$$
t_{1}=\varphi_{1}^{-1}\left(T_{1}\right), T_{1} \in(T, T+U) .
$$

Next, we obtain from (9.4) by (9.9) $\left(t_{1} \rightarrow \infty \Leftrightarrow T \rightarrow \infty\right)$

$$
t_{1}-T_{1}=\mathcal{O}\left(\frac{t_{1}}{\ln t_{1}}\right) \Rightarrow 1-\frac{T_{1}}{t_{1}}=\mathcal{O}\left(\frac{1}{\ln t_{1}}\right) \rightarrow 0, T \rightarrow \infty,
$$

i.e.

$$
t_{1} \sim T_{1} \sim T, T \rightarrow \infty
$$

and (see (9.10), (9.11) 


$$
t_{1}-T=t_{1}-T_{1}+T_{1}-T=\mathcal{O}\left(\frac{t_{1}}{\ln t_{1}}\right)+\mathcal{O}(U)=\mathcal{O}\left(\frac{T}{\ln T}\right),
$$

where $U \leq \frac{T}{\ln T}$ by the condition of the Lemma 4. Now, (see (9.12) )

$$
\ln t_{1}=\ln T+\mathcal{O}\left(\frac{t_{1}-T}{T}\right)=\ln T+\mathcal{O}\left(\frac{U}{T}\right)=\ln T+\mathcal{O}\left(\frac{1}{\ln T}\right) .
$$

Then the formula (9.7) follows from (9.8) by (9.1), (9.13).

\section{Proof of Theorem 2}

10.1. Putting

$$
f(t)=\left|\zeta\left(\frac{1}{2}+i t\right)\right|^{4}
$$

into (9.6) we obtain

$$
\int_{T}^{T+U_{1}}\left|\zeta\left(\frac{1}{2}+i \varphi_{1}(t)\right)\right|^{4}\left|\zeta\left(\frac{1}{2}+i t\right)\right|^{2} \mathrm{~d} t \sim \ln T \int_{\varphi_{1}(T)}^{\varphi_{1}\left(T+U_{1}\right)} Z^{4}(t) \mathrm{d} t,
$$

i.e. we have to consider the integral (see 8.2 )

$$
\begin{aligned}
& \int_{\varphi_{1}(T)}^{\varphi_{1}\left(T+U_{1}\right)} Z^{4}(t) \mathrm{d} t= \\
& \left\{\varphi_{1}(t) \sum_{k=0}^{4} C_{k} \ln ^{4-k} \varphi_{1}(t)\right\}_{t=T}^{t=T+U_{1}}+\mathcal{O}\left(T^{7 / 8+\epsilon}\right)= \\
& \sum_{k=0}^{4} C_{k} V_{k}+\mathcal{O}\left(T^{7 / 8+\epsilon}\right)
\end{aligned}
$$

where

$$
V_{k}=\varphi_{1}\left(T+U_{1}\right) \ln ^{4-k} \varphi_{1}\left(T+U_{1}\right)-\varphi_{1}(T) \ln ^{4-k} \varphi_{1}(T),
$$

and, for example,

$$
\begin{aligned}
& V_{0}=\varphi_{1}\left(T+U_{1}\right) \ln ^{4} \varphi_{1}\left(T+U_{1}\right)-\varphi_{1}(T) \ln ^{4} \varphi_{1}(T)= \\
& =\left.\left[\varphi_{1}\left(T+U_{1}\right)-\varphi_{1}(T)\right] \frac{\mathrm{d}}{\mathrm{d} \varphi_{1}}\left[\varphi_{1}(t) \ln ^{4}\left(\varphi_{1}(t)\right)\right]\right|_{t=d_{0}}= \\
& =\left[\varphi_{1}\left(T+U_{1}\right)-\varphi_{1}(T)\right]\left[\ln ^{4} \varphi_{1}\left(d_{0}\right)+4 \ln ^{3} \varphi_{1}\left(d_{0}\right)\right]= \\
& =U_{1} \frac{\varphi_{1}\left(T+U_{1}\right)-\varphi_{1}(T)}{U_{1}} \ln ^{4} \varphi_{1}\left(d_{0}\right)\left\{1+\mathcal{O}\left(\frac{1}{\ln \varphi_{1}\left(d_{0}\right)}\right)\right\}, \\
& \varphi_{1}\left(d_{0}\right) \in\left(\varphi_{1}(T), \varphi_{1}\left(T+U_{1}\right)\right) .
\end{aligned}
$$


10.2. By the Ingham formula (see [4, p. 294, [10, p. 120)

$$
\int_{0}^{T} Z^{2}(t) \mathrm{d} t=T \ln T+(2 c-1-\ln 2 \pi) T+\mathcal{O}\left(T^{1 / 2} \ln T\right)
$$

we have in the case $U_{1}=T^{7 / 8+2 \epsilon}$

$$
\int_{T}^{T+U_{1}} Z^{2}(t) \mathrm{d} t=U_{1} \ln T+(2 c-\ln 2 \pi) U_{1}+\mathcal{O}\left(T^{7 / 8+\epsilon}\right) .
$$

Comparing the formulae (2.5) and (10.5) we obtain

$$
\frac{\varphi_{1}\left(T+U_{1}\right)-\varphi_{1}(T)}{U_{1}}=\tan \left[\alpha\left(T, U_{1}\right)\right]=1+\mathcal{O}\left(\frac{\ln \ln T}{\ln T}\right) .
$$

Since (see (10.6)

$$
\varphi_{1}\left(d_{0}\right)-\varphi_{1}(T) \leq \varphi_{1}\left(T+U_{1}\right)-\varphi_{1}(T)=\mathcal{O}\left(U_{1}\right),
$$

we have (see (9.4); $\varphi_{1}(T) \sim T$ )

$$
\begin{aligned}
& \ln \varphi_{1}\left(d_{0}\right)=\ln \varphi_{1}(T)+\ln \left\{1+\frac{\varphi_{1}\left(d_{0}\right)-\varphi_{1}(T)}{\varphi_{1}(T)}\right\}= \\
& =\ln \varphi_{1}(T)+\mathcal{O}\left(\frac{U_{1}}{T}\right) \sim \ln T .
\end{aligned}
$$

Hence, we obtain from (10.4) by (10.6), (10.7)

$$
V_{0} \sim U_{1} \ln ^{4} T
$$

and similarly

$$
V_{l}=\mathcal{O}\left(U_{1} \ln ^{4-l} T\right), l=1,2,3,4
$$

Finally, the formula (8.5) follows from (10.1) by (10.2), (10.8) and (10.9).

\section{JACOB'S LADDERS AND A NEW CLASS OF THE NONLINEAR INTEGRAL EQUATIONS; CONCLUDING REMARKS}

11.1. The proof of the Theorem 2 is simultaneously the proof of the following theorem.

Theorem 3. Every Jacob's ladder $\varphi_{1}(t)=\frac{1}{2} \varphi(t)$, where $\varphi(t)$ is the exact solution of the nonlinear integral equation

$$
\int_{0}^{\mu[x(T)]} Z^{2}(t) e^{-\frac{2}{x(T)} t} \mathrm{~d} t=\int_{0}^{T} Z^{2}(t) \mathrm{d} t
$$

is the asymptotic solution of the following nonlinear integral equation

$$
\int_{T}^{T+U_{1}}\left|\zeta\left(\frac{1}{2}+i x(t)\right)\right|^{4}\left|\zeta\left(\frac{1}{2}+i t\right)\right|^{2} \mathrm{~d} t=\frac{1}{2 \pi^{2}} U_{1} \ln ^{5} T, U_{1}=T^{7 / 8+2 \epsilon}
$$

Page 13 of 16 
where $x(t)=x(t ; T, \epsilon)$, for every fixed $T \geq T_{0}[\varphi]$, i.e. the following asymptotic formula (see 8.5)

$$
\frac{2 \pi^{2}}{U_{1} \ln ^{5} T} \int_{T}^{T+U_{1}}\left|\zeta\left(\frac{1}{2}+i \varphi_{1}(t)\right)\right|^{4}\left|\zeta\left(\frac{1}{2}+i t\right)\right|^{2} \mathrm{~d} t \sim 1, T \rightarrow \infty
$$

holds true.

11.2. Let us remind the Selberg's formula ( 9 , p. 128)

$$
\int_{T}^{T+U_{2}}\{S(t)\}^{2 k} \mathrm{~d} \sim \frac{(2 k) !}{k !(2 \pi)^{2 k}} U_{2}(\ln \ln T)^{k},
$$

where $U_{2}=T^{1 / 2+\epsilon}$, and $k$ is the fixed positive number, and

$$
S(t)=\frac{1}{\pi} \arg \zeta\left(\frac{1}{2}+i t\right)
$$

(where the arg is defined by the usual way). From (11.2) by (9.7) one obtains

$$
\begin{aligned}
& \int_{\varphi_{1}^{-1}(T)}^{\varphi_{1}^{-1}\left(T+U_{2}\right)}\left\{\arg \zeta\left(\frac{1}{2}+i \varphi_{1}(t)\right)\right\}^{2 k}\left|\zeta\left(\frac{1}{2}+i t\right)\right|^{2} \mathrm{~d} t \sim \\
& \sim \frac{(2 k) !}{k !(2)^{2 k}} U_{2} \ln T(\ln \ln T)^{k}, T \rightarrow \infty .
\end{aligned}
$$

Remark 14. This formula cannot be obtained in the classical theory of A. Selberg and, all the less, in the theories of Balasubramanian, Heath-Brown and Ivic.

Some nonlocal interaction of the functions

$$
\left\{\arg \zeta\left(\frac{1}{2}+i \varphi_{1}(t)\right)\right\}^{2 k},\left|\zeta\left(\frac{1}{2}+i t\right)\right|^{2}
$$

is expressed by the formula (11.3).

Remark 15. Every Jacob's ladder $\varphi_{1}(t)$ is the asymptotic solution (see (11.3) ) of the nonlinear integral equation

$$
\begin{aligned}
& \int_{x^{-1}(T)}^{x^{-1}\left(T+U_{2}\right)}\left\{\arg \zeta\left(\frac{1}{2}+i x(t)\right)\right\}^{2 k}\left|\zeta\left(\frac{1}{2}+i t\right)\right|^{2} \mathrm{~d} t= \\
& =\frac{(2 k) !}{k ! 2^{2 k}} U_{2} \ln T(\ln \ln T)^{k}
\end{aligned}
$$

11.3. Since

$$
\int_{T}^{T+U} \pi(x) \mathrm{d} x \sim \frac{U T}{\ln T}, U \leq \frac{T}{\ln T}
$$

holds true, we obtain (see (9.7), $f(t)=\pi(t)$ )

$$
\int_{\varphi_{1}^{-1}(T)}^{\varphi_{1}^{-1}(T+U)} \pi\left[\varphi_{1}(t)\right]\left|\zeta\left(\frac{1}{2}+i t\right)\right|^{2} \mathrm{~d} t \sim \frac{U T}{\ln T},
$$

where $t-\varphi_{1}(t) \sim(1-c) \pi(t)$. 
Remark 16. Every Jacob's ladder $\varphi_{1}(t)$ is the asymptotic solution (see (11.5)) of the following nonlinear integral equation

$$
\int_{x^{-1}(T)}^{x^{-1}(T+U)} \pi[x(t)]\left|\zeta\left(\frac{1}{2}+i t\right)\right|^{2} \mathrm{~d} t=\frac{U T}{\ln T}, 0<U \leq \frac{T}{\ln T} .
$$

11.4. Another source of the integrals containing the function $\left|\zeta\left(\frac{1}{2}+i t\right)\right|^{2}$ is, for example, the system of the Chebyshev polynomials $\mathcal{T}_{n}(x), x \in[-1,1], n=0,1,2, \ldots$ of the first kind. We obtain, from the well-known formula

$$
\int_{-1}^{1} \frac{\left[\mathcal{T}_{n}(x)\right]^{2}}{\sqrt{1-x^{2}}} \mathrm{~d} x=\left\{\begin{array}{lll}
\frac{\pi}{2} & , & n \geq 1 \\
\pi & , & n=0
\end{array}\right.
$$

the following one

$$
\int_{T}^{T+2} \frac{\left[\mathcal{T}_{n}(t-T-1)\right]^{2}}{\sqrt{1-(t-T-1)^{2}}} \mathrm{~d} t=\frac{\pi}{2}, n \geq 1
$$

Next, putting

$$
f(t)=\frac{\left[\mathcal{T}_{n}(t-T-1)\right]^{2}}{\sqrt{1-(t-T-1)^{2}}}
$$

into (9.7), $\stackrel{\circ}{T}=\varphi_{1}^{-1}(T), \widehat{T+2}=\varphi_{1}^{-1}(T+2)$, we obtain

$$
\int_{\varphi_{1}^{-1}(T)}^{\varphi_{1}^{-1}(T+2)} \frac{\left[\mathcal{T}_{n}\left(\varphi_{1}(t)-T-1\right)\right]^{2}}{\sqrt{1-\left(\varphi_{1}(t)-T-1\right)^{2}}}\left|\zeta\left(\frac{1}{2}+i t\right)\right|^{2} \mathrm{~d} t \sim \frac{\pi}{2} \ln T, n \geq 1 .
$$

Remark 17. Jacob's ladder $\varphi_{1}(t)$ is the asymptotic solution of the nonlinear integral equation (see (11.2))

$$
\int_{x^{-1}(T)}^{x^{-1}(T+2)} \frac{\left[\mathcal{T}_{n}(x(t)-T-1)\right]^{2}}{\sqrt{1-\left(\varphi_{1}(t)-T-1\right)^{2}}}\left|\zeta\left(\frac{1}{2}+i t\right)\right|^{2} \mathrm{~d} t=\frac{\pi}{2} \ln T, n \geq 1 .
$$

11.5. Let us remind the Liapunov equation

$$
\gamma \int_{(a)} \frac{\rho^{\prime}\left(\mathrm{d} x^{\prime}\right)^{3}}{\left|\vec{r}-\vec{r}^{\prime}\right|}+\frac{1}{2} \omega_{i k} \omega_{j k}\left(x_{i}-a_{i}\right)\left(x_{j}-a_{j}\right)=V_{a}
$$

(comp. 11, pp. 334-337) for determining of the form of the integration domain (a), (the density $\rho$ is prescribed), i.e. the equilibrium figures of the rotating body.

Analogically to the case (11.9), we will call the segment $\left[x^{-1}(T), x^{-1}(T+2)\right]$ entering the equation (11.8), for example, the equilibrium segment and the segment $\left[\varphi_{1}^{-1}(T), \varphi_{1}^{-1}(T+2)\right]$ will be called the asymptotical equilibrium segment.

Remark 18. By (11.7) there is, for every fixed $T \geq T_{0}[\varphi]$, a continuum of the asymptotic equilibrium segments $\left[\varphi_{1}^{-1}(T), \varphi_{1}^{-1}(T+2)\right]$. However, is there any equilibrium segment $\left[x^{-1}(T), x^{-1}(T+2)\right]$ for some $T \geq T_{0}[\varphi]$ ? 
Remark 19. There are the fixed-point methods and other methods of the functional analysis used to study the nonlinear equations. What can be obtained by using these methods in the case of the nonlinear integral equations of the type (11.1), (11.4), (11.6), (11.8)?

I would like to thank Ekatherina Karatsuba and Michal Demetrian for their moral support of my study of the Jacob's ladders.

\section{REFERENCES}

[1] V.A. Fock, 'The theory of space, time and gravitation', GITTL, Moscow, 1955, (in russian).

[2] G.H. Hardy and J.E. Littlewood, 'Contributions to the theory of the Riemann zeta-function and the theory of the distribution of primes', Acta Math. 41 (1), (1918), 119-196.

[3] G.H. Hardy and J.E. Littlewood, 'The approximate functional equation in the theory of the zeta-function with applications to the divisor problems of Dirichlet and Piltz', Proc. Lond. Math. Soc. (2) 21, (1922), 39-74.

[4] A.E. Ingham, 'Mean-value theorems in the theory of the Riemann zeta-function', Proc. Lond. Math. Soc. (2), 27, (1926), 273-300.

[5] A. Ivic, 'The Riemann zeta-function', A Willey-Interscience Publications, New York, 1985.

[6] A.A. Karatsuba, 'Complex Analysis in Number Theory', CRC Press, Boca Raton, Ann Arbor, London, Tokyo, 1995.

[7] J.E. Littlewood, 'Two notes on the Riemann zeta-function', Proc. Cam. Phil. Soc., 22 (1924), 234-242.

[8] J. Moser, 'Jacob's ladders and the almost exact asymptotic representation of the HardyLittlewood integral', Math. Notes 2010, 88, pp. 414-422.

[9] A. Selberg, 'Contributions to the theory of the Riemann zeta-function', Arch. for Math. og Naturv. B 48, (1946), 89-155.

[10] E.C. Titchmarsh, 'The theory of the Riemann zeta-function' Clarendon Press, Oxford, 1951.

Department of Mathematical Analysis and Numerical Mathematics, Comenius University, Mlynska Dolina M105, 84248 Bratislava, SLOVAKIA

E-mail address: jan.mozer@fmph.uniba.sk 